\title{
PRIMER HALLAZGO DEL GÉNERO SYSCENUS HARGER, 1880 (ISOPODA, CYMOTHOIDA, AEGIDAE), EN EL GOLFO DE MÉXICO, CON LA DESCRIPCIÓN DE UNA ESPECIE NUEVA
}

\author{
Carlos Varela
}

Department of Marine Biology and Ecology, Rosenstiel School of Marine and Atmospheric Science (R. S. M. A. S.), University of Miami.varela06@gmail.com

\section{RESUMEN}

Se describe una especie nueva de isópodo de aguas profundas perteneciente al género Syscenus. Esta es la primera cita de una especie de este género para el Golfo de México. Se presenta además una clave para identificar las especies pertenecientes a este género.

Palabras clave: Isopoda, Cymothoida, Aegidae, Syscenus, Golfo de México.

Title: First finding of the genus Syscenus Harger, 1880 (Isopoda, Cymothoida, Aegidae) in the Gulf of Mexico, with the description of a new species.

\section{ABSTRACT}

A new species of deep water isopod of the genus Syscenus is described. This is the first record of a species of this genus for the Gulf of Mexico. A key for the identification of all the species of the genus is also presented.

Keywords: Isopoda, Cymothoida, Aegidae, Syscenus, Gulf of Mexico.

\section{INTRODUCCIÓN}

Hasta el presente, solo ocho especies pertenecientes a la familia Aegidae White, 1850 han sido halladas en el Golfo de México; cinco en el género Aega Leach, 1815 y tres en el género Rocinela Leach, 1818. Todas estas especies han sido colectadas entre 55 y 2200 metros de profundidad (Schotte et al., 2009). El género Syscenus Harger, 1880 consta actualmente de nueve especies, de las cuales se conocen siete en el Océano Pacífico y solo dos han sido halladas en el OcéanoAtlántico (Bruce, 2009).

Estudio de material perteneciente a la familia Aegidae depositado en el Museo de Invertebrados Marinos de Rosenstiel School of Marine and Atmospheric Science (R. S. M. A. S.) permitió encontrar una especie del género Syscenus aún no descrita. El ejemplar fue colectado en el límite Este del Golfo de México (Felder et al., 2009), siendo esta la primera cita de Syscenus para las aguas de dicha zona y la especie descrita en este trabajo es la tercera del género en el Océano Atlántico.

\section{OBJETIVO}

- Describir una nueva especie de isópodo de aguas profundas del género Syscenus. 


\title{
MATERIALES Y METODOS
}

El material estudiado fue producto de los viajes de exploración del buque de investigación de la Universidad de Miami, B/I Gerda en los cayos de Florida, Estados Unidos de América (E. U. A.). Las muestras fueron obtenidas usando una rastra Otter. El ejemplar de Syscenus fue estudiado bajo el microscopio estereoscópico y las ilustraciones se realizaron con un microscopio clínico con cámara lucida acoplada. El material ha sido depositado en el Museo de Invertebrados Marinos de R. S. M. A. S.

\author{
RESULTADOS \\ Familia Aegidae \\ Syscenus kensleyi sp. nov.
}

(Figuras 1-3)

Diagnosis. Ojos ausentes; lámina frontal más ancha que larga; flagelo de la antena se extiende hasta el pereionito 6; coxas 4-7 visibles en vista dorsal; coxas de los pereonitos 2-7 con el margen posteroventral aguzado; pleonito 5 sin espinas dorsales, pleotelson casi tan largo como ancho (0.8: 1$)$, posteriormente redondeado y ramas del urópodo sin setas robustas.

Diagnosis. Eyes absent; frontal lamina wider than long; antennal flagellum reaching the sixth somite; coxae of pereonites 2-7 with posteroventral margen pointed; pleonite 5 without dorsal spines; coxae 4-7 visible in dorsal view; pleotelson almost longer than wide (0.8: 1), rounded posteriorly and rami of the uropod without robust setae.

Descripción del holotipo. Hembra adulta, no ovígera, de $38.8 \mathrm{~mm}$ de largo y $14.2 \mathrm{~mm}$ de ancho, 2.7 veces tan larga como ancha, superficie dorsal con aspecto liso y pulido, ancho máximo a nivel del pereonito 5. Rostro estrecho anteriormente, en vista dorsal se ve truncado y se dobla hacia debajo. Ojos ausentes. (Fig. 1A).

Cefalón (Fig. 1K) con el rostro doblado hacia debajo entre la base de las antenas, margen anterolateral levemente sinuoso; largo de los pereonitos, medidos en su mitad dorsal, $1>2>3=4<5<6>7$; las coxas no se extienden mas alla del final de su respectivo pereonito, coxas 2 y 4 con el ángulo posteroventral aguzado y 5-7 posteriormente aguzadas, coxas 4-7 visibles en vista dorsal (Fig. 1B).

Pleón, con el pleonito 1 estrecho, casi oculto por el pereonito 7; pleonitos 2-5 subsimilares en longitud; pleonito 4 con los márgenes posterolaterales que no se extienden hasta los márgenes posteriores del pleonito 5; pleonito 5 con los ángulos posterolaterales agudos (Fig. 1B). Pleotelson (Fig. 1J) con el largo que es 0.8 del ancho máximo, con márgenes laterales convexos; superficie dorsal lisa sin depresiones submediales, margen posterior convexo con una escotadura a cada lado del extremo distal, que es ancho y redondeado.

Lámina frontal (Fig. 1I) más ancha que larga (0.9: 1), con un proceso redondeado anteriormente, márgenes laterales cóncavos y margen posterior ligeramente convexo.

Mandíbula (Fig. 1C) con el incisivo agudo y esclerotizado, separado del molar, palpo con tres artejos subiguales, artejo 2 con setas en su porción distal, artejo 3 curvado con 28 setas que se distribuyen a lo largo del margen lateral y van aumentando de longitud hacia el extremo distal. Maxila 1 (Fig. 1F), delgada con cinco setas distales robustas. Maxila 2 (Fig. 1D) cerca de dos veces el ancho de la maxila 1, con lóbulos pobremente definidos distalmente, apicalmente con tres setas robustas curvadas. Maxilípedo (Fig. 1E) con el artejo 1 rectangular y alargado, desarmado, artejo 2 muy corto, con dos setas robustas y la porción terminal del artejo 3 con una seta robusta. 

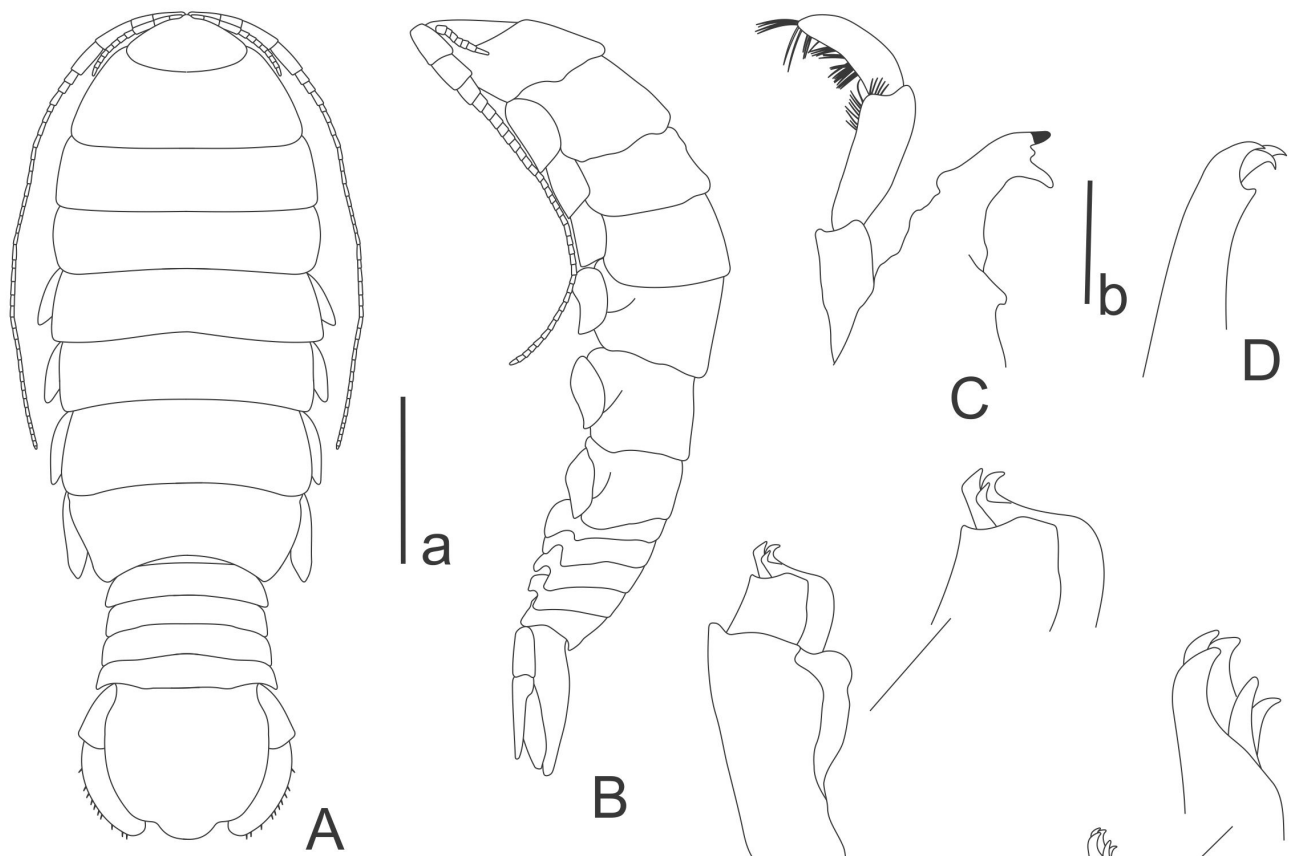

C
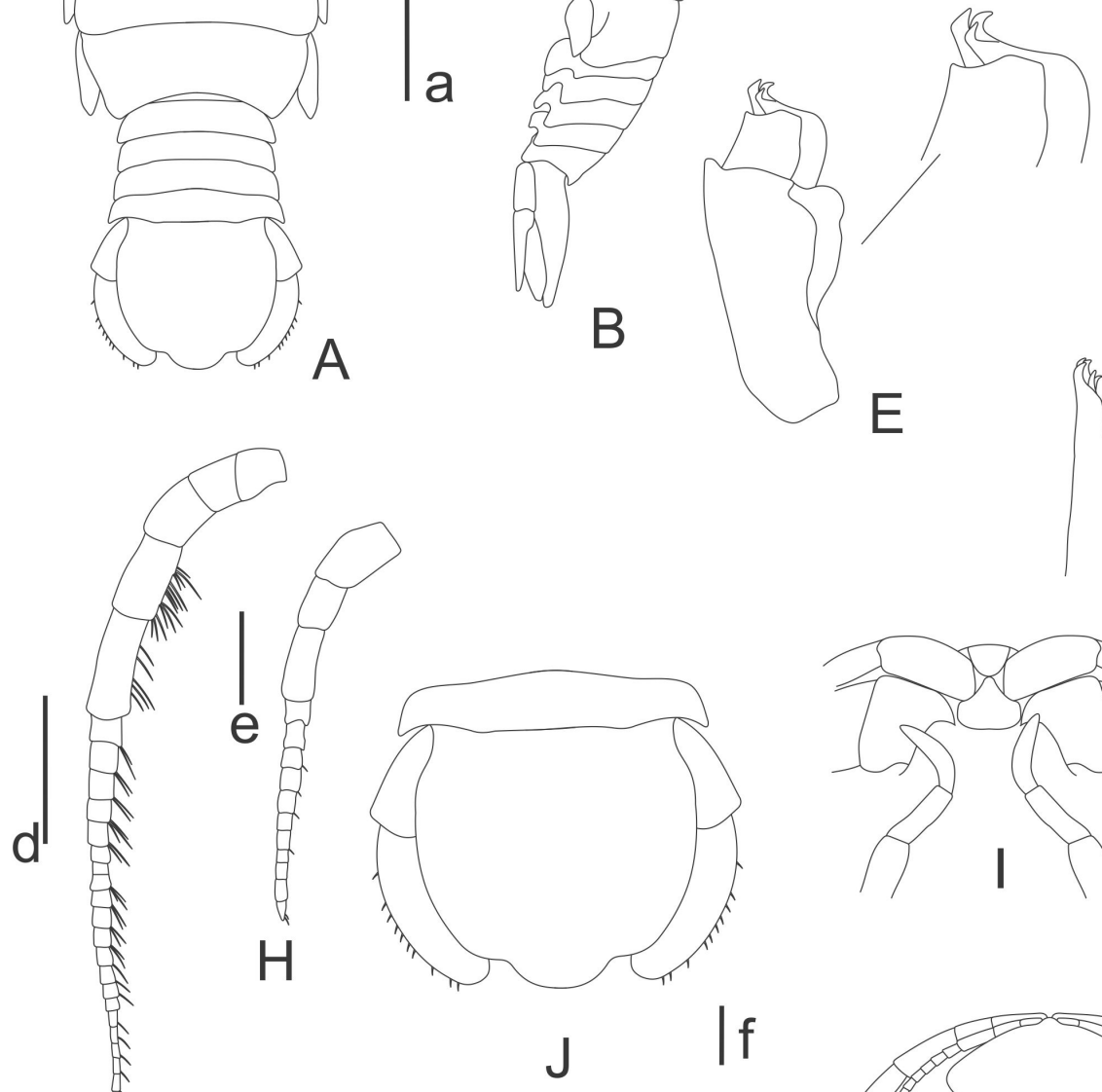

G
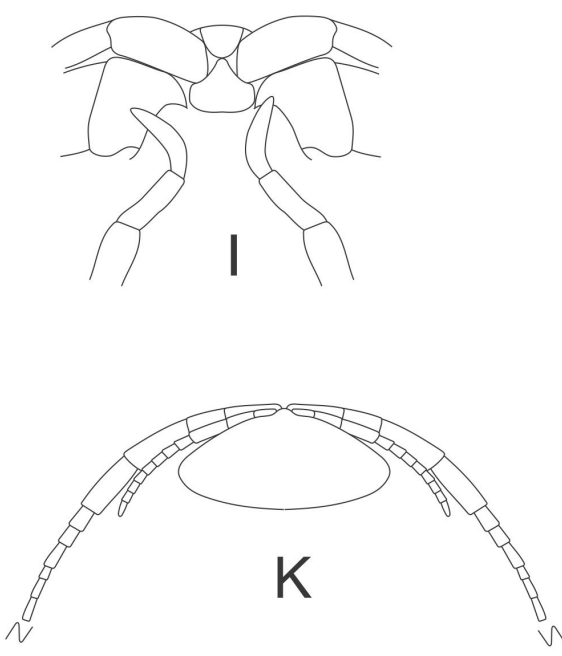

Figura 1, A-K. A, Vista dorsal del holotipo (a). B, Vista lateral del holotipo (a). C, Mandíbula (b). D, Maxila (b). E, Maxilípedo (b). F, Maxílula (c). G, Anténula (d). H, Antena (e). I, Lámina frontal (c). J, Pleotelson (f). K, Cabeza (f). Escala: a, 10 mm; b y c, $0,5 \mathrm{~mm}$; d y f, $5 \mathrm{~mm}$ y e, $2 \mathrm{~mm}$. 
Antena (Fig. 1G) con los artejos 1 y 2 cortos, subiguales; artejo 3, 1.3 veces más largo que el artejo 2; artejo 4, 1.2 veces el largo del artejo 3; artejo 5 es 1.5 veces el largo que el artejo 4; flagelo con 41 artejos. Anténula ( Fig. 1H) alcanza el artejo 5 de la antena; artejo 1 subigual en longitud al artejo 3 pero casi dos veces más ancho, separados dorsalmente por el rostro y ventralmente por la lámina frontal; artejo 2, 0.6 del largo del artejo 3; flagelo con 11 artejos.

Pereópodo 1 (Fig. 2A) con basipodito 2.5 veces tan largo como su mayor ancho, isquiopodito 2.5 veces tan largo como ancho, margen interior sin setas robustas, margen distal superior con dos setas, meropodito con margen inferior sin setas robustas, margen distal superior con tres setas, carpopodito 1.6 veces tan largo como el meropodito, margen inferior sin setas robustas, propodito 2.2 veces tan largo como su ancho proximal, palma del propodito simple, sin procesos ni setas, margen inferior sin setas robustas, dactilopodito tan largo como el propodito. Pereópodos 2 (Fig. 2B) y 3 (Fig. 2C) similiares al pereiópodo 1.

Pereópodo 4 (Fig. 2D) con basipodito 2.8 veces tan largo como su máximo ancho, margen inferior sin setas; isquiopodito 0.5 veces tan largo como el basipodito, margen inferior con una seta robusta, ángulo distal superior con siete setas robustas, ángulo distal inferior con cuatro setas robustas, meropodito 0.6 veces tan largo como el isquiopodito, casi tan largo como ancho, margen inferior con una seta robusta, ángulo distal superior con nueve setas robustas, ángulo distal inferior con dos setas robustas; carpopodito 1.8 veces tan largo como ancho 0.8 veces más largo que el isquiopodito, margen inferior con una seta robusta, ángulo distal superior con 5 setas robustas y ángulo distal inferior con cuatro setas robustas; propodito 0.9 veces tan largo como el isquiopodito, 2.5 veces tan largo como ancho, margen inferior con una seta robusta, ángulo distal sin setas, ángulo distal superior sin setas, ángulo distal inferior con una seta robusta. Dactilopodito curvado y aguzado, casi del mismo largo que el propodito.

Pereópodo 5 (Fig. 2E) con basipodito 2.4 veces tan largo como su máximo ancho, margen inferior sin setas; isquiopodito 0.7 veces tan largo como el basipodito, margen inferior con una seta robusta, ángulo distal superior con cinco setas robustas y tres setas simples, ángulo distal inferior con cinco setas robustas, meropodito 0.6 veces tan largo como el isquiopodito, 1.8 veces tan largo como ancho, margen inferior con una seta robusta; ángulo distal superior con 14 setas, cinco de ellas robustas; ángulo distal inferior con cinco setas robustas; carpopodito1.8 veces tan largo como ancho 0.7 veces más largo que el isquiopodito margen inferior con una seta robusta, ángulo distal superior con cuatro setas, dos de ellas robustasy ángulo distal inferior con cuatro setas robustas; propodito 0.8 veces tan largo como el isquiopodito, tres veces tan largo como ancho, margen inferior con una seta robusta,ángulo distal superior con una seta robusta, ángulo distal inferior con dos setas robustas. Dactilopodito curvado y aguzado, casi del mismo largo que el propodito.

Pereópodo 6 (Fig. 2F) con basipodito 2.8 veces tan largo como su máximo ancho, margen inferior sin setas; isquiopodito 0.7 veces tan largo como el basipodito, margen inferior con dossetas robustas, ángulo distal superior con ochosetas robustas, ángulo distal inferior con cinco setas robustas; meropodito 0.6 veces tan largo como el isquiopodito, 1.6 veces tan largo como ancho, margen inferior con una seta robusta; ángulo distal superior con siete setas robustas, ángulo distal inferior con cuatro setas robustas; carpopodito 3.5 veces tan largo como ancho, 0.6 veces más largo que el isquiopodito, margen inferior con dos setas robustas, ángulo distal superior con seis setas robustas y ángulo distal inferior con cinco setas robustas; propodito 0.7 veces tan largo como el isquiopodito, 3.5 veces tan largo como ancho, margen inferior con una seta robusta, ángulo distal superior sin setas, ángulo distal inferior con una seta robusta. Dactilopodito curvado y aguzado, 0.8 del largo del propodito. 

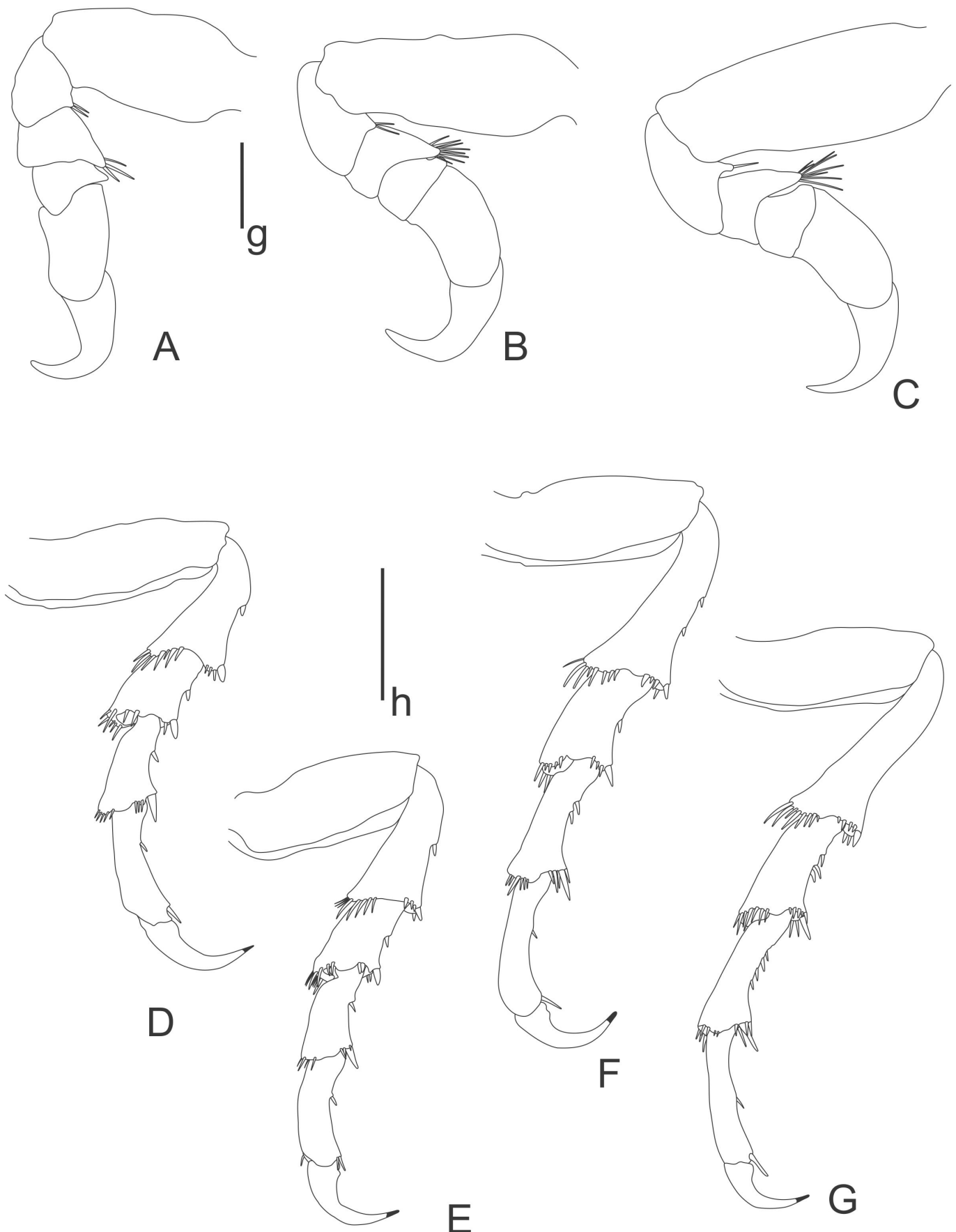

E
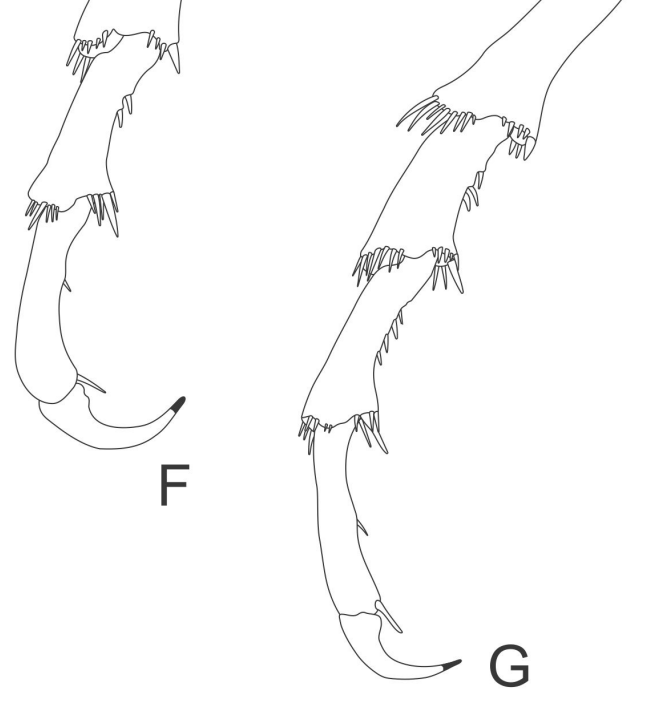

Figura 2, A-G. A, Pereópodo 1 (a). B, Pereópodo 2 (a). C, Pereópodo 3 (a). D, Pereópodo 4 (b). E, Pereópodo 5 (b). F, Pereópodo 6 (b). G, Pereópodo 7 (b). Escala: g y h, $3 \mathrm{~mm}$. 
Pereópodo 7 (Fig. 2G) con basipodito 2.5 veces tan largo como su máximo ancho, margen inferior sin setas; isquiopodito casi tan largo como el basipodito, margen inferior sin setas robustas, ángulo distal superior con ocho setas robustas, ángulo distal inferior con siete setas robustas, meropodito 0.5 veces tan largo como el isquiopodito, 2.4 veces tan largo como ancho, margen inferior con tres setas robustas, ángulo distal superior con siete setas robustas, ángulo distal inferior con ocho setas robustas; carpopodito 3.5 veces tan largo como ancho, 0.7 veces más largo que el isquiopodito, margen inferior con cuatro setas robustas, ángulo distal superior con cinco setas robustas y ángulo distal inferior con seis setas robustas, propodito 0.6 veces tan largo como el isquiopodito, 5.9 veces tan largo como ancho, margen inferior con una seta robusta, ángulo distal sin setas, ángulo distal superior sin setas, ángulo distal inferior con una seta robusta. Dactilopodito curvado y aguzado, 0.8 del largo del propodito.

Pleópodo 1 (Fig. 3A) con protopodito rectangular, con 11 setas plumosas en el margen mesial, ramas subiguales en longitud, el exopodito es un tercio el ancho del endopodito. Pleópodo 2 (Fig. 3B) protopodito rectangular, con cerca de 12 setas plumosas en el margen mesial, exopodito más ampliamente redondeado que el endopodito, 1.5 veces el ancho del endopodito. Pleópodo 3 (Fig. 3C) con protopodito rectangular con cerca de 12 setas plumosas en el margen mesial, exopodito subcircular, más ancho que el endopodito. Pleópodo 4 (Fig. 3D) con protopodito rectangular, con 15 setas plumosas en el margen mesial con exopodito subcircular más ancho que el endopodito. Pleópodo 5 (Fig. 3E) con protopodito rectangular, con seis setas plumosas en el margen mesial con protopodito estrecho, exopodito subcircular pero más pequeño que el exopodito.

Protopodito del urópodo alargado mesiodistalmente, exopodito estrechamente ovado, es 0.8 de la longitud del endopodito, ampliamente redondeado distalmente. Ambas ramas con finas setas en los márgenes (Fig. 3, F y G).

Tipos. Holotipo: hembra no ovígera, E. U. A. Colectada al sur de los cayos de La Florida, en la

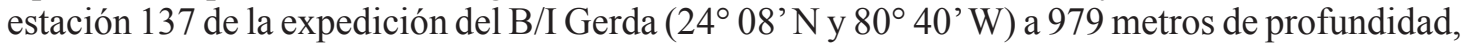
el 22. vi. 1963. Depositado en la Coleccion de Invertebrados Marinos de R.S.M.A.S. UMML 32.9314.

Comentario. Syscenus kensleyi, especie nueva, no presenta ojos; pleotelson posteriormente redondeado; pleonito 5 sin espinas dorsales y ramas del urópodo sin setas robustas. Dentro del género solo S. kapoo Bruce, 2009, de Nueva Zelanda, S. intermedius Richardson, 1901, del Mar de China e Indonesia y S. atlanticus Kononenko, 1988, del Atlántico Norte comparten estos caracteres.

La especie nueva Syscenus kensleyi, presenta el margen posterior del pleotelson convexo con una escotadura a cada lado del extremo distal, que es ancho y redondeado; lámina frontal más ancha que larga y el flagelo de la antena se extiende hasta el pereonito 6; mientras que $S$. kapoo no presenta escotaduras en el extremo distal del pleotelson; lámina frontal es más larga que ancha y el flagelo de la antena alcanza el pereonito 3. S. atlanticus presenta el pleotelson dos veces más ancho que largo; coxas de los pereionitos 2-7 redondeadas posteriormente y lámina frontal triangular con el margen posterior convexo; mientras que $S$. kensleyi, especie nueva, el pleotelson el largo es menos de la mitad del ancho; las coxas de los pereonitos 2-7 son puntiagudas proteriormente y la lámina frontal no es triangular. En $S$. intermedius las ramas del urópodo son casi de igual longitud; el flagelo antenal llega hasta el pereonito 4 y las coxas son redondeadas, mientras que en $S$. kensleyi, especie nueva, las ramas del urópodo son desiguales en longitud; el flagelo antenal llega hasta el sexto pereonito y el margen posteroventral de las coxas es aguzado. 

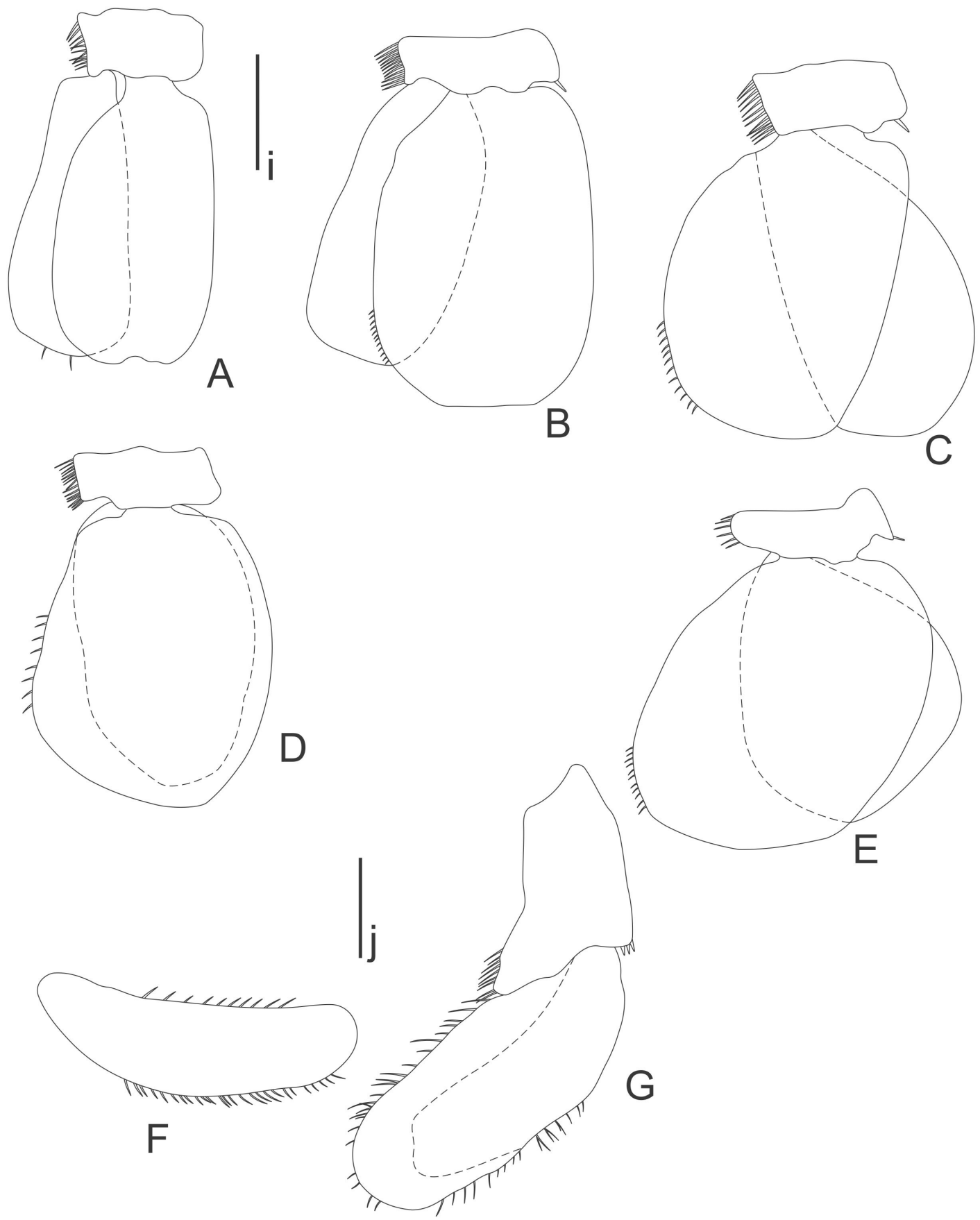

Figura 3, A-G. A, Pleópodo 1 (a). B, Pleópodo 2 (a). C, Pleópodo 3 (a). D, Pleópodo 4 (a). E, Pleópodo 5 (a). F, Urópodo (b). $\mathrm{G}$, Vista interna del urópodo (b). Escala: i y j, $3 \mathrm{~mm}$. 
Distribución. Solo se conoce para la localidad tipo.

Etimología. La especie está dedicada al Dr. Brian Kensley, por su valiosa contribución al conocimiento de los isópodos marinos.

\section{CLAVE PARA LA IDENTIFICACIÓN DE LAS ESPECIES PERTENECIENTES AL GÉNERO SYSCENUS}

1a- Especies con ojos 2

1b- Especies sin ojos 3

2a- Ojos bien desarrollados, protopodito del pleópodo 5 provisto de ganchos de acoplamiento. Pereiópodo 7 con basipodito y meropodito subiguales en tamaño.... Syscenus karu Bruce, 2005 (Vanuatu, Océano Pacífico). 2b-Ojos degenerados, carecen de pigmento pero presentan ommatídeos, protopodito del pleópodo 5 sin ganchos de acoplamiento. Pereiópodo 7 con basipodito y meropodito de tamaños diferentes Syscenus peruanus Menzies y George, 1972 (Perú, Océano Pacífico).

3a- Pleotelson aguzado posteriormente 4

3b- Pleotelson subtruncado o redondeado posteriormente 5

4a- Urópodos no se extienden más allá del margen posterior del pleotelson; pleotelson ligeramente curvado y aguzado posteriormente Syscenus sprinthorpei Bruce, 1997

(Australia, OcéanoPacífico).

4b- Urópodos se extienden más allá del margen posterior del pleotelson; pleotelson con márgenes cóncavos y aguzado posteriormente. Syscenus infelix Harger, 1880 (Atlántico Norte y Mar Mediterráneo).

5a- Ambas ramas del urópodo con setas robustas Syscenus moana Bruce, 2005 (Nueva Caledonia, Océano Pacífico).

$5 b$ - Ambas ramas del urópodo sin setas robustas

6a- Pleotelson dos veces más ancho que largo Syscenus atlanticus Kononenko, 1985 (Atlántico Norte).

6b- Pleotelson casi tan largo como ancho 7

7a- Pleonito 5 con una espina dorsal Syscenus latus Richardson, 1909 (Japón, Nueva Zalanda y Nueva Caledonia, Océano Pacífico).

7b- Pleonito 7 sin espinas dorsales 8 
8a- Pleotelson con extermo distal redondeado, con una escotadura a cada lado, flagelo de la antena llega hasta el pereonito 6 Syscenus kensleyi, especie nueva

(Cayos de La Florida, Océano Atlántico). 8b- Pleotelson con el extremo distal redondeado, sin escotaduras en sus lados, flagelo de la antena no llega hasta el pereonito 6

9a- Ramas del urópodo casi de igual longitud; flagelo antenal llega hasta el pereonito 4; ángulo posteroventral de las coxas redondeado. Syscenus intermedius Richardson, 1910 (Mar de China e Indonesia, Océano Pacífico). 9b- Exopodito del urópodo más largo que el endopodito; flagelo antenal llega hasta el pereonito 3; ángulo posteroventral de las coxas aguzado. Syscenus kapoo Bruce, 2009

(Nueva Zelanda, Océano Pacífico).

\section{AGRADECIMIENTOS}

Queremos agradecer a la Profesora Emérita de Investigación Nancy Voss, Curadora del Museo de Invertebrados Marinos de R. S. M. A. S. (Universidad de Miami), por habernos permitido el estudio del material depositado en la colección y por su ayuda incondicional. Al revisor anónimo que con sus sugerencias ayudaron a mejorar el trabajo.

\section{REFERENCIAS}

Bruce, N. L. 2009. The marine Fauna of New Zealand: Isopoda, Aegidae (Crustacea). NIWA Biodiversity Memoir, 122: 1-252.

Felder, D. L., D. K. Camp y J. W Tunnell Jr. 2009. 1. An Introduction to Gulf of Mexico Biodiversity Assesment. En: Gulf of Mexico origin, water and biota (J. W. Tunnell, D. Felder y S. Earle eds).1-14.

Schotte, M., J. C. Markham y G. D. F. Wilson. 2009. 55. Isopoda (Crustacea) of the Gulf of Mexico. En: Gulf of Mexico origin, water and biota (J. W. Tunnell, D. Felder y S. Earle eds), 973-986.

[Recibido: 26 de febrero, 2015. Aceptado para publicación: 22 de junio, 2015] 